\title{
Yield performance of new juice grape varieties grafted onto different rootstocks under tropical conditions
}

\author{
Marlon Jocimar Rodrigues da Silva, ${ }^{\mathrm{a}, *}$, Ana Paula Maia Paiva ${ }^{\mathrm{a}}$, Adilson Pimentel Junior ${ }^{\mathrm{a}}$, \\ Camilo André Pereira Contreras Sánchez ${ }^{\mathrm{a}}$, Daniel Callili ${ }^{\mathrm{a}}$, Mara Fernandes Moura ${ }^{\mathrm{b}}$, \\ Sarita Leonel ${ }^{\mathrm{a}}$, Marco Antonio Tecchio ${ }^{\mathrm{a}}$ \\ a São Paulo State University (Unesp), School of Agriculture, José Barbosa de Barros Street, $n^{\circ}$ 1780, 18.610-307, Botucatu, SP, Brazil \\ ${ }^{\mathrm{b}}$ Instituto Agronômico (IAC), Centro APTA de Frutas, Luiz Pereira dos Santos Avenue, $n^{o} 1.500$, CEP 13214-820, Jundiaí, SP, Brazil
}

A R T I C L E I N F O

\section{Keywords:}

Vitis labrusca L.

Hybrid grapes

Grafting

Grape juice

Tropical viticulture

\begin{abstract}
A B S T R A C T
The objective of this work was to evaluate the yield components and physicochemical characteristics of bunches and berries of new grape varieties for juice elaboration, which were 'Isabel Precoce' (Vitis labrusca L.) and the hybrids 'BRS Carmem', 'BRS Cora' and IAC 138-22 'Máximo' grown onto 'IAC 572' and 'IAC 766' rootstocks under tropical conditions. The yield components (number of bunches and yield per vine, as well as productivity) and the physicochemical characteristics of the bunches and berries of the eight scion-rootstock combinations were evaluated in three seasons. All data were subjected to analysis of variance and principal components analysis. The varieties 'Isabel Precoce', 'BRS Cora' and IAC 13822 'Máximo' produced high fruit yield, with the number of bunches and yield per vine similar to one another and superior to those of 'BRS Carmem'. Significant differences occurred among varieties in the physicochemical grape characteristics. 'Isabel Precoce' and 'BRS Carmem' grapes had balanced levels of sugar and acid content, and 'BRS Cora' presented large bunches and berries, reaching high soluble solids content despite the high acidity. IAC 138-22 'Máximo' grape also had large bunches but small berries and limited potential in the accumulation of sugars. The 'IAC 766' rootstock resulted in the best performance across all four varieties evaluated, showing maximum results in terms of fruit yield and physicochemical quality attributes of grapes.
\end{abstract}

\section{Introduction}

Grapes are one of the world's most commonly produced fruit crops. In addition to the increasing consumption of fresh grapes, in recent years, interest in products made from grapes has greatly increased, particularly in grape juices (FAO and OIV, 2017). The numerous health benefits may be part of the reason because these products are rich sources of phenolic compounds (phytochemicals with potential antioxidant activity) (Granato et al., 2016).

Almost any type of grape variety can produce grape juice. However, technological and economic constraints limit the varieties used. Some varieties are particularly dedicated to grape juice, such as 'Concord' (Vitis labrusca L.), which is cultivated in the United States of America and Brazil (FAO and OIV, 2017). However, in Brazil, the 'Isabel' ( $V$. labrusca) is the primary raw material used for the elaboration of grape juices, despite limitations. 'Isabel' grapes confer low color intensity and low bioactive compounds content to grape juice (Lima et al., 2014). Additionally, these $V$. labrusca varieties are mostly planted in the subtropical and temperate areas of the country (Mello, 2017).

To meet the demands of producing grape juices in tropical conditions worldwide, breeding programs have been developing new interspecific hybrid grape varieties ( $V$. vinifera $\times V$. labrusca). The goal is to develop varieties that, in addition to having suitable characteristics for adapting to warmer environments, show high yield capacity, tolerance to the primary fungal diseases and produce grapes with good quality for juice production (Camargo and Maia, 2004). Thus, in addition to the 'Isabel Precoce', a spontaneous somatic mutation of the 'Isabel' (Camargo, 2004), the hybrid varieties 'BRS Carmem', 'BRS Cora' and IAC 138-22 'Máximo' have been recently released.

In some tropical areas, because of the climate, more than one crop a year can be produced with high temperatures and a defined rainy season in the summer, the dry winter and the use of budburst stimulators. Therefore, programming the pruning according to demands of industry is possible (Camargo et al., 2012).

Several factors can influence grapevine yield and grape quality, which include the use of grafting. Extensively used in viticulture,

\footnotetext{
* Corresponding author.

E-mail address: marlon.silva@fca.unesp.br (M.J.R.d. Silva).
} 
grafting is an effective technique for controlling soilborne diseases and for overcoming abiotic stresses (Ibacache et al., 2016; Jin et al., 2016). Additionally, many studies report that different rootstocks affect the duration of phenological stages, canopy structure, growth, yield and fruit quality (Bascunán-Godoy et al., 2017; Koundouras et al., 2008; Silva et al., 2017).

The scion-rootstock relationship is extremely specific, depending on affinity and compatibility of the combination and the soil and climatic adaptation (Vrsic et al., 2015). Thus, the ideal scion-rootstock combination is very important in a production system. Currently, in tropical conditions, varieties of $V$. vinifera table grapes are cultivated onto 'IAC 572', considered of high vigor. However, with the introduction of new hybrids and $V$. labrusca grape varieties intended for juice production, further studies are required to achieve useful scientific data for use in rootstock recommendations for these varieties.

In this context, the objective of this work was to evaluate the yield components and physicochemical characteristics of bunches and berries of new juice grape varieties ( $V$. labrusca and hybrids) grown onto 'IAC 572' and 'IAC 766' rootstocks under tropical conditions.

\section{Materials and methods}

\subsection{Experimental location and growing conditions}

The study was conducted in an experimental vineyard located in Votuporanga, São Paulo State, Brazil (latitude $20^{\circ} 20^{\prime} \mathrm{S}$, longitude $49^{\circ} 58^{\prime} \mathrm{W}$; elevation $525 \mathrm{~m}$ ), during three consecutive seasons performed within two years (2015-2016). According to the Köppen classification, the climate is type Aw, i.e., tropical climate with dry winter. An automatic meteorological station (Campbell Scientific ${ }^{\circ}$, Logan, UT, EUA) installed in the experimental area recorded meteorological conditions during the study period. The mean temperature was $24.1^{\circ} \mathrm{C}$, the minimum average was $16.6^{\circ} \mathrm{C}$, and the maximum average was $31.7^{\circ} \mathrm{C}$. The average annual rainfall was $1495 \mathrm{~mm}$, with a tendency for concentrated rainfall in the summer months. The soil was classified as Argissolo Vermelho-Amarelo (equivalent to Ultisol, USDA soil taxonomy) according to previously published criteria (Embrapa, 2006).

The experiment was established in the winter of 2013 when the rootstocks were planted, and the scions were grafted in the winter of 2014. The vines were trained on a unilateral cordon system ( $1 \mathrm{~m}$ above the soil) in a vertical shoot positioning by means of iron wires and spaced $2.0 \times 1.1 \mathrm{~m}$ apart (4545 vines per hectare).

During the trials, all the cultural practices regarding fertilization, weed control, and pest and disease management were conducted as standard regional cultivation practices. The entire vineyard was covered with polyethylene screen with $18 \%$ shading, to protect against bird attack. The irrigation of the plants was performed with a micro sprinkler system, as described by Conceição et al. (2017).

Regular crop pruning was performed in February and August 2015 and July 2016, and the grapes were harvested in June and December 2015 and December 2016, respectively. For all seasons, vines were cane pruned to leave one to two nodes, as well the usual cultivation practices in the region. Subsequently, $5 \%$ hydrogen cyanamide was applied to the buds to induce and standardize the sprouting.

\subsection{Treatments and experimental design}

One Vitis labrusca L., 'Isabel Precoce', and three hybrid grape varieties, 'BRS Carmem' (Muscat Belly A $\times$ H 65.9.14), 'BRS Cora' (Muscat Belly A $\times$ H. 65.9.14) and IAC 138-22 'Máximo' (Seibel $11342 \times$ Syrah), were grown onto the rootstocks 'IAC 766' Campinas (106-8 Mgt $\times V$. caribaea $)$ and 'IAC 572' Jales (V. caribaea $\times 101-14 \mathrm{Mgt})$.

A completely randomized block design in a two-factor arrangement (factorial scheme $4 \times 2$ ) with five replicates was used as the statistical model. The factors evaluated consisted of four scions and two rootstocks. The plot consisted of four vines, with a total of 20 vines per treatment (scion-rootstock combination).

\subsection{Harvest and measurements}

The plots were harvested at full maturity stage of the grapes. At harvest, the number of bunches per vine and their masses were recorded to estimate the yield per vine $(\mathrm{kg})$ and productivity $\left(\mathrm{t} \mathrm{ha}^{-1}\right)$.

The physical measurements of the grapes were assessed by determining the bunch mass $(\mathrm{g})$, length $(\mathrm{cm})$ and width $(\mathrm{cm})$ in a sample of 10 bunches per plot collected at harvest of each season, which were also used to measure the rachis mass ( $\mathrm{g}$ ). For berry mass (g), length $(\mathrm{cm})$ and width $(\mathrm{cm}), 10$ berries were collected from each bunch, totaling 100 berries per plot. The number of berries per bunch was estimated using the relation [(bunch mass - rachis mass)/berry mass]. The ratio rachis mass/bunch mass was calculated and expressed as a percentage.

For the chemical evaluations of the berries, $\mathrm{pH}$, soluble solids (SS), titratable acidity (TA), maturation index (SS/TA) and reducing sugars were determined. The $\mathrm{pH}$ of the grape must was determined directly by a potentiometer (Tecnal ${ }^{\circ}$, Piracicaba, SP, Brazil). The SS was determined using a digital refractometer with automatic temperature compensation (Reichert ${ }^{\circ}, r^{2} i 300$ model, Buffalo, NY, EUA), and the result is expressed in units of ${ }^{\circ} \mathrm{Brix}$. TA was determined through the titration of the grape must with a $0.1 \mathrm{~N} \mathrm{NaOH}$ solution in a semi-automatic titrator, adopting the end-point at $\mathrm{pH}=8.2$, and the result is expressed in percent of tartaric acid (OIV, 2011). The reducing sugars were determined according to the Somogy-Nelson colorimetric method (Nelson, 1944). The absorbance values at $535 \mathrm{~nm}$ were compared with those of a calibration curve of glucose in a UV/vis spectrophotometer (BEL Photonics ${ }^{\circ}$, Piracicaba, SP, Brazil), and the results are expressed in percent of glucose.

\subsection{Statistical analysis}

Means of the three seasons were subjected to analysis of variance (two-way ANOVA) to determine the effect of scions and rootstocks and their interaction, and then means were compared by Tukey's test $(p<$ 0.05) using the SISVAR version 5.4 statistical program (Lavras, MG, Brazil). Additionally, data of the eight scion-rootstocks, including 17 traits, were analyzed via XLSTAT statistical software version 19.4 (Addinsoft, NY, USA). Principal component analysis (PCA) was applied to all productive and physicochemical attributes, which were also evaluated by Pearson's correlation.

\section{Results and discussion}

All data represent an average of three seasons performed within two years $(2015 / 2016)$. Statistically significant variations were not detected among the seasons.

No significant interaction $(p>0.05)$ was detected between scions and rootstocks for all yield components (Table 1) and physicochemical characteristics evaluated (Tables 2 and 3). Thus, both factors (scions and rootstocks) were analyzed separately.

\subsection{Yield components}

Analyzing the isolated effect of the canopy varieties on the yield components, 'Isabel Precoce', 'BRS Cora' and IAC 138-22 'Máximo' presented a number of bunches that were similar to one another, with an average of 26 bunches per vine. This value was approximately 2.3fold higher than that found on 'BRS Carmem' vines (Table 1).

The number of bunches per vine was the variable that most contributed to the yield, which was verified by the high positive and significant correlation $(r)$ between those characteristics $(r=0.91, p<$ 0.01; data not shown). Similar results are found by others for $V$. vinifera grape varieties (Bascunán-Godoy et al., 2017; Ibacache et al., 2016). 
Table 1

Number of bunches per vine, yield and productivity of grape varieties grown onto different rootstocks under tropical conditions.

\begin{tabular}{|c|c|c|c|c|c|c|c|c|c|}
\hline \multirow{2}{*}{$\begin{array}{l}\text { Yield components } \\
\text { (unit) }\end{array}$} & \multirow[t]{2}{*}{ Season $^{1}$} & \multicolumn{5}{|l|}{ Scion } & \multicolumn{3}{|l|}{ Rootstock } \\
\hline & & $\begin{array}{l}\text { Isabel } \\
\text { Precoce }\end{array}$ & BRS Carmem & BRS Cora & $\begin{array}{l}\text { IAC 138-22 } \\
\text { Máximo }\end{array}$ & $p$-value & IAC 766 & IAC 572 & $p$-value \\
\hline \multirow[t]{4}{*}{ Number of bunches per vine } & I & $32.4 \pm 5.2$ & $13.1 \pm 5.8$ & $22.3 \pm 6.9$ & $45.1 \pm 9.3$ & & $28.8 \pm 14.9$ & $27.7 \pm 12.9$ & \\
\hline & II & $19.3 \pm 7.8$ & $9.8 \pm 3.6$ & $15.7 \pm 6.3$ & $8.4 \pm 3.6$ & & $17.1 \pm 7.4$ & $9.4 \pm 4.0$ & \\
\hline & III & $32.4 \pm 9.7$ & $11.0 \pm 4.5$ & $32.9 \pm 12.8$ & $25.3 \pm 5.8$ & & $29.2 \pm 13.8$ & $21.6 \pm 9.7$ & \\
\hline & $\mathrm{Av}$ & $28.0 \pm 5.9 \mathrm{a}$ & $11.3 \pm 3.3 \mathrm{~b}$ & $23.6 \pm 6.4 \mathrm{a}$ & $26.3 \pm 3.5 \mathrm{a}$ & $<0.01$ & $25.0 \pm 8.7 \mathrm{a}$ & $19.6 \pm 6.7 b$ & $<0.01$ \\
\hline \multirow[t]{4}{*}{ Yield per vine (kg) } & I & $2.99 \pm 0.7$ & $1.63 \pm 0.6$ & $2.16 \pm 0.6$ & $3.78 \pm 1.2$ & & $3.07 \pm 1.3$ & $2.21 \pm 0.8$ & \\
\hline & II & $2.40 \pm 1.1$ & $1.28 \pm 0.6$ & $1.99 \pm 1.0$ & $0.99 \pm 0.6$ & & $2.30 \pm 0.9$ & $1.04 \pm 0.6$ & \\
\hline & III & $3.31 \pm 1.2$ & $1.45 \pm 0.7$ & $2.36 \pm 1.0$ & $3.10 \pm 0.8$ & & $3.02 \pm 1.3$ & $2.09 \pm 0.9$ & \\
\hline & $\mathrm{Av}$ & $2.90 \pm 0.9 \mathrm{a}$ & $1.45 \pm 0.4 \mathrm{c}$ & $2.17 \pm 0.7 \mathrm{~b}$ & $2.63 \pm 0.7 \mathrm{ab}$ & $<0.01$ & $2.80 \pm 0.9 \mathrm{a}$ & $1.78 \pm 0.5 b$ & $<0.01$ \\
\hline \multirow{4}{*}{$\begin{array}{l}\text { Productivity } \\
\qquad\left(\mathrm{t} \mathrm{ha}^{-1}\right)\end{array}$} & I & $13.6 \pm 3.1$ & $7.4 \pm 2.7$ & $9.8 \pm 2.7$ & $17.2 \pm 5.5$ & & $13.9 \pm 5.9$ & $10.0 \pm 3.6$ & \\
\hline & II & $10.9 \pm 5.0$ & $5.8 \pm 3.0$ & $9.1 \pm 4.5$ & $4.5 \pm 2.8$ & & $10.4 \pm 4.3$ & $4.7 \pm 2.7$ & \\
\hline & III & $15.0 \pm 5.7$ & $6.6 \pm 3.2$ & $10.7 \pm 4.7$ & $14.1 \pm 3.5$ & & $13.7 \pm 5.7$ & $9.5 \pm 4.1$ & \\
\hline & $\mathrm{Av}$ & $13.2 \pm 4.2 \mathrm{a}$ & $6.6 \pm 1.9 c$ & $9.9 \pm 3.4 b$ & $11.9 \pm 3.0 \mathrm{ab}$ & $<0.01$ & $12.7 \pm 4.1 \mathrm{a}$ & $8.1 \pm 2.2 \mathrm{~b}$ & $<0.01$ \\
\hline
\end{tabular}

Average values (Av) are reported as the mean \pm standard deviation $(n=5)$ from three seasons. Different letters within a row for each factor (Scion and Rootstock) indicate a significant difference according to Tukey's test $(p<0.05)$.

1 I, II and III: measurements performed at first, second and third seasons, respectively.

Table 2

Physical attributes of bunches and berries of grape varieties grown onto different rootstocks under tropical conditions.

\begin{tabular}{|c|c|c|c|c|c|c|c|c|c|}
\hline \multirow{2}{*}{$\begin{array}{l}\text { Physical traits } \\
\text { (unit) }\end{array}$} & \multirow[t]{2}{*}{ Season $^{1}$} & \multicolumn{5}{|l|}{ Scion } & \multicolumn{3}{|l|}{ Rootstock } \\
\hline & & $\begin{array}{l}\text { Isabel } \\
\text { Precoce }\end{array}$ & BRS Carmem & BRS Cora & $\begin{array}{l}\text { IAC 138-22 } \\
\text { Máximo }\end{array}$ & $p$-value & IAC 766 & IAC 572 & $p$-value \\
\hline \multirow{4}{*}{$\begin{array}{l}\text { Bunch mass } \\
\text { (g) }\end{array}$} & I & $126.4 \pm 25$ & $198.9 \pm 41$ & $123.4 \pm 26$ & $122.1 \pm 18$ & & $152.7 \pm 45$ & $132.7 \pm 39$ & \\
\hline & II & $128.7 \pm 17$ & $133.2 \pm 43$ & $147.6 \pm 32$ & $123.7 \pm 33$ & & $150.1 \pm 25$ & $116.5 \pm 32$ & \\
\hline & III & $116.9 \pm 20$ & $129.5 \pm 31$ & $101.1 \pm 21$ & $156.5 \pm 27$ & & $133.6 \pm 31$ & $118.4 \pm 30$ & \\
\hline & Av & $124.0 \pm 20 \mathrm{~b}$ & $153.9 \pm 31 \mathrm{a}$ & $124.0 \pm 21 b$ & $134.1 \pm 14 \mathrm{ab}$ & $<0.01$ & $145.5 \pm 23 a$ & $122.5 \pm 21 b$ & $<0.01$ \\
\hline \multirow[t]{4}{*}{ Bunch length $(\mathrm{cm})$} & I & $10.3 \pm 1.0$ & $13.0 \pm 1.0$ & $12.6 \pm 1.0$ & $11.8 \pm 0.9$ & & $12.1 \pm 0.8$ & $11.7 \pm 1.8$ & \\
\hline & II & $9.7 \pm 0.8$ & $10.7 \pm 1.3$ & $12.5 \pm 1.3$ & $10.8 \pm 1.6$ & & $11.6 \pm 1.2$ & $10.3 \pm 1.7$ & \\
\hline & III & $11.3 \pm 0.6$ & $11.0 \pm 1.2$ & $11.9 \pm 1.0$ & $13.2 \pm 1.5$ & & $11.9 \pm 1.5$ & $11.8 \pm 1.2$ & \\
\hline & Av & $10.43 \pm 0.7 \mathrm{c}$ & $11.57 \pm 0.7 \mathrm{~b}$ & $12.33 \pm 0.7 \mathrm{a}$ & $11.92 \pm 0.8 \mathrm{ab}$ & $<0.01$ & $11.87 \pm 0.9 \mathrm{a}$ & $11.25 \pm 1.0 \mathrm{~b}$ & $<0.01$ \\
\hline \multirow{4}{*}{$\begin{array}{l}\text { Bunch width } \\
(\mathrm{cm})\end{array}$} & I & $6.5 \pm 0.5$ & $7.5 \pm 0.5$ & $8.0 \pm 0.8$ & $6.5 \pm 0.5$ & & $7.1 \pm 0.7$ & $7.1 \pm 1.1$ & \\
\hline & II & $6.0 \pm 0.3$ & $6.5 \pm 0.7$ & $6.7 \pm 0.7$ & $6.1 \pm 0.9$ & & $6.6 \pm 0.7$ & $6.0 \pm 0.6$ & \\
\hline & III & $5.8 \pm 0.4$ & $6.3 \pm 0.6$ & $7.6 \pm 0.8$ & $8.4 \pm 1.1$ & & $7.2 \pm 1.3$ & $6.8 \pm 1.3$ & \\
\hline & Av & $6.07 \pm 0.3 c$ & $6.75 \pm 0.4 b$ & $7.42 \pm 0.5 \mathrm{a}$ & $7.00 \pm 0.5 \mathrm{ab}$ & $<0.01$ & $6.97 \pm 0.6 \mathrm{a}$ & $6.65 \pm 0.6 \mathrm{~b}$ & 0.016 \\
\hline \multirow[t]{4}{*}{ Number of berries per bunch } & I & $42.8 \pm 5.0$ & $74.1 \pm 11.2$ & $38.4 \pm 6.3$ & $81.4 \pm 13.8$ & & $60.4 \pm 21.4$ & $57.9 \pm 21.5$ & \\
\hline & II & $36.1 \pm 5.5$ & $46.1 \pm 11.8$ & $46.1 \pm 9.2$ & $68.5 \pm 19.0$ & & $56.1 \pm 18.9$ & $42.3 \pm 11.5$ & \\
\hline & III & $35.2 \pm 4.5$ & $74.3 \pm 4.5$ & $32.0 \pm 6.4$ & $98.9 \pm 13.4$ & & $61.8 \pm 29.2$ & $58.5 \pm 30.1$ & \\
\hline & Av & $38.02 \pm 4.7 \mathrm{c}$ & $64.85 \pm 5.7 b$ & $38.83 \pm 5.3 c$ & $82.94 \pm 8.2 \mathrm{a}$ & $<0.01$ & $59.43 \pm 21.1 \mathrm{a}$ & $52.89 \pm 18.7 \mathrm{~b}$ & $<0.01$ \\
\hline \multirow{4}{*}{$\begin{array}{l}\text { Berry mass } \\
\quad(\mathrm{g})\end{array}$} & I & $2.86 \pm 0.2$ & $2.56 \pm 0.2$ & $3.14 \pm 0.3$ & $1.44 \pm 0.1$ & & $2.62 \pm 0.7$ & $2.38 \pm 0.6$ & \\
\hline & II & $3.47 \pm 0.2$ & $2.79 \pm 0.2$ & $3.12 \pm 0.1$ & $1.72 \pm 0.1$ & & $2.79 \pm 0.7$ & $2.75 \pm 0.7$ & \\
\hline & III & $3.24 \pm 0.1$ & $2.83 \pm 0.2$ & $3.09 \pm 0.2$ & $1.51 \pm 0.1$ & & $2.72 \pm 0.7$ & $2.61 \pm 0.6$ & \\
\hline & Av & $3.19 \pm 0.2 \mathrm{a}$ & $2.73 \pm 0.1 \mathrm{~b}$ & $3.12 \pm 0.2 \mathrm{a}$ & $1.56 \pm 0.1 \mathrm{c}$ & $<0.01$ & $2.71 \pm 0.7 \mathrm{a}$ & $2.58 \pm 0.6 \mathrm{~b}$ & $<0.01$ \\
\hline \multirow{4}{*}{$\begin{array}{l}\text { Berry length } \\
\text { (cm) }\end{array}$} & I & $1.90 \pm 0.1$ & $1.82 \pm 0.1$ & $2.01 \pm 0.1$ & $1.44 \pm 0.0$ & & $1.83 \pm 0.2$ & $1.75 \pm 0.2$ & \\
\hline & II & $1.99 \pm 0.1$ & $1.93 \pm 0.1$ & $2.04 \pm 0.0$ & $1.55 \pm 0.0$ & & $1.88 \pm 0.2$ & $1.88 \pm 0.2$ & \\
\hline & III & $1.90 \pm 0.0$ & $1.86 \pm 0.1$ & $1.96 \pm 0.1$ & $1.45 \pm 0.0$ & & $1.82 \pm 0.2$ & $1.77 \pm 0.2$ & \\
\hline & Av & $1.93 \pm 0.1 \mathrm{~b}$ & $1.87 \pm 0.1 \mathrm{c}$ & $2.00 \pm 0.1 \mathrm{a}$ & $1.48 \pm 0.1 \mathrm{~d}$ & $<0.01$ & $1.84 \pm 0.2 \mathrm{a}$ & $1.80 \pm 0.2 \mathrm{~b}$ & $<0.01$ \\
\hline \multirow{4}{*}{$\begin{array}{l}\text { Berry width } \\
\quad(\mathrm{cm})\end{array}$} & I & $1.61 \pm 0.1$ & $1.55 \pm 0.0$ & $1.64 \pm 0.1$ & $1.26 \pm 0.0$ & & $1.54 \pm 0.2$ & $1.49 \pm 0.1$ & \\
\hline & II & $1.73 \pm 0.0$ & $1.62 \pm 0.0$ & $1.64 \pm 0.0$ & $1.35 \pm 0.0$ & & $1.58 \pm 0.1$ & $1.59 \pm 0.1$ & \\
\hline & III & $1.68 \pm 0.0$ & $1.63 \pm 0.0$ & $1.63 \pm 0.0$ & $1.30 \pm 0.0$ & & $1.56 \pm 0.2$ & $1.55 \pm 0.1$ & \\
\hline & $\mathrm{Av}$ & $1.67 \pm 0.0 \mathrm{a}$ & $1.60 \pm 0.0 \mathrm{c}$ & $1.64 \pm 0.1 \mathrm{~b}$ & $1.30 \pm 0.1 \mathrm{~d}$ & $<0.01$ & $1.56 \pm 0.2 \mathrm{a}$ & $1.54 \pm 0.2 \mathrm{~b}$ & 0.014 \\
\hline \multirow{4}{*}{$\begin{array}{l}\text { Rachis mass } \\
\text { (g) }\end{array}$} & I & $4.06 \pm 0.8$ & $6.37 \pm 1.3$ & $3.34 \pm 0.7$ & $5.86 \pm 1.2$ & & $5.15 \pm 1.7$ & $4.66 \pm 1.6$ & \\
\hline & II & $3.91 \pm 0.8$ & $3.86 \pm 1.3$ & $3.26 \pm 0.7$ & $6.35 \pm 1.8$ & & $4.98 \pm 1.9$ & $3.71 \pm 1.2$ & \\
\hline & III & $3.16 \pm 0.4$ & $3.01 \pm 0.7$ & $2.40 \pm 0.5$ & $7.47 \pm 1.6$ & & $4.17 \pm 2.1$ & $3.85 \pm 2.3$ & \\
\hline & $\mathrm{Av}$ & $3.71 \pm 0.6 \mathrm{bc}$ & $4.41 \pm 0.9 \mathrm{~b}$ & $3.00 \pm 0.5 c$ & $6.56 \pm 0.8 \mathrm{a}$ & $<0.01$ & $4.77 \pm 1.6 \mathrm{a}$ & $4.07 \pm 1.4 \mathrm{~b}$ & $<0.01$ \\
\hline \multirow[t]{4}{*}{ Ratio rachis mass/bunch mass (\%) } & I & $3.25 \pm 0.4$ & $3.24 \pm 0.4$ & $2.72 \pm 0.2$ & $4.75 \pm 0.5$ & & $3.46 \pm 0.9$ & $3.53 \pm 0.8$ & \\
\hline & II & $3.03 \pm 0.3$ & $3.08 \pm 0.3$ & $2.42 \pm 0.3$ & $5.41 \pm 0.4$ & & $3.48 \pm 1.2$ & $3.49 \pm 1.2$ & \\
\hline & III & $2.74 \pm 0.3$ & $2.35 \pm 0.2$ & $2.38 \pm 0.1$ & $4.74 \pm 0.3$ & & $3.04 \pm 1.0$ & $3.07 \pm 1.1$ & \\
\hline & Av & $3.01 \pm 0.1 \mathrm{~b}$ & $2.89 \pm 0.1 \mathrm{~b}$ & $2.51 \pm 0.1 \mathrm{c}$ & $4.97 \pm 0.3 \mathrm{a}$ & $<0.01$ & $3.33 \pm 1.0 \mathrm{a}$ & $3.36 \pm 1.0 \mathrm{a}$ & 0.487 \\
\hline
\end{tabular}

Average values (Av) are reported as the mean \pm standard deviation $(n=5)$ from three seasons. Different letters within a row for each factor (Scion and Rootstock) indicate a significant difference according to Tukey's test $(p<0.05)$.

${ }^{1}$ I, II and III: measurements performed at first, second and third seasons, respectively. 
Table 3

Chemical attributes of bunches and berries of grape varieties grown onto different rootstocks under tropical conditions.

\begin{tabular}{|c|c|c|c|c|c|c|c|c|c|}
\hline \multirow{2}{*}{$\begin{array}{l}\text { Chemical traits } \\
\text { (unit) }\end{array}$} & \multirow[t]{2}{*}{ Season $^{1}$} & \multicolumn{5}{|l|}{ Scion } & \multicolumn{3}{|l|}{ Rootstock } \\
\hline & & $\begin{array}{l}\text { Isabel } \\
\text { Precoce }\end{array}$ & BRS Carmem & BRS Cora & $\begin{array}{l}\text { IAC 138-22 } \\
\text { Máximo }\end{array}$ & $p$-value & IAC 766 & IAC 572 & $p$-value \\
\hline \multirow[t]{4}{*}{$\mathrm{pH}$} & I & $3.13 \pm 0.0$ & $3.07 \pm 0.1$ & $3.09 \pm 0.0$ & $3.33 \pm 0.1$ & & $3.14 \pm 0.1$ & $3.17 \pm 0.1$ & \\
\hline & II & $3.29 \pm 0.1$ & $3.36 \pm 0.2$ & $3.20 \pm 0.0$ & $3.27 \pm 0.1$ & & $3.25 \pm 0.1$ & $3.31 \pm 0.1$ & \\
\hline & III & $3.30 \pm 0.0$ & $3.69 \pm 0.2$ & $3.23 \pm 0.0$ & $3.38 \pm 0.1$ & & $3.38 \pm 0.2$ & $3.42 \pm 0.2$ & \\
\hline & $\mathrm{Av}$ & $3.24 \pm 0.0 \mathrm{~b}$ & $3.37 \pm 0.1 \mathrm{a}$ & $3.17 \pm 0.0 c$ & $3.33 \pm 0.1 \mathrm{a}$ & $<0.01$ & $3.26 \pm 0.1 \mathrm{~b}$ & $3.30 \pm 0.1 \mathrm{a}$ & 0.013 \\
\hline \multirow[t]{4}{*}{ SS $\left({ }^{\circ}\right.$ Brix $)$} & I & $14.1 \pm 0.5$ & $14.7 \pm 0.5$ & $14.4 \pm 0.5$ & $12.1 \pm 0.3$ & & $13.8 \pm 1.2$ & $13.8 \pm 1.1$ & \\
\hline & II & $14.6 \pm 0.7$ & $14.5 \pm 0.7$ & $15.2 \pm 0.6$ & $14.0 \pm 0.8$ & & $14.8 \pm 0.8$ & $14.4 \pm 0.8$ & \\
\hline & III & $17.5 \pm 0.9$ & $16.1 \pm 0.9$ & $18.1 \pm 0.2$ & $14.4 \pm 1.0$ & & $16.9 \pm 1.5$ & $16.2 \pm 1.7$ & \\
\hline & $\mathrm{Av}$ & $15.40 \pm 0.5 \mathrm{ab}$ & $15.08 \pm 0.5 b$ & $15.90 \pm 0.3 \mathrm{a}$ & $13.51 \pm 0.5 \mathrm{c}$ & $<0.01$ & $15.14 \pm 0.9 \mathrm{a}$ & $14.80 \pm 1.1 \mathrm{~b}$ & 0.019 \\
\hline \multirow[t]{4}{*}{ TA (\%) } & I & $1.12 \pm 0.1$ & $1.35 \pm 0.2$ & $1.78 \pm 0.1$ & $1.15 \pm 0.1$ & & $1.39 \pm 0.3$ & $1.31 \pm 0.3$ & \\
\hline & II & $0.75 \pm 0.1$ & $0.71 \pm 0.1$ & $1.19 \pm 0.1$ & $0.80 \pm 0.1$ & & $0.88 \pm 0.2$ & $0.85 \pm 0.3$ & \\
\hline & III & $0.64 \pm 0.0$ & $0.65 \pm 0.2$ & $0.92 \pm 0.0$ & $0.63 \pm 0.0$ & & $0.72 \pm 0.1$ & $0.70 \pm 0.1$ & \\
\hline & $\mathrm{Av}$ & $0.84 \pm 0.1 \mathrm{~b}$ & $0.90 \pm 0.1 \mathrm{~b}$ & $1.30 \pm 0.0 \mathrm{a}$ & $0.86 \pm 0.0 \mathrm{~b}$ & $<0.01$ & $1.00 \pm 0.2 \mathrm{a}$ & $0.95 \pm 0.2 \mathrm{~b}$ & 0.025 \\
\hline \multirow[t]{4}{*}{ SS/TA } & I & $12.7 \pm 1.4$ & $11.1 \pm 2.1$ & $8.2 \pm 0.8$ & $10.5 \pm 0.6$ & & $10.2 \pm 1.6$ & $11.0 \pm 2.5$ & \\
\hline & II & $19.5 \pm 1.9$ & $21.4 \pm 5.5$ & $12.8 \pm 0.9$ & $17.7 \pm 2.1$ & & $17.3 \pm 2.9$ & $18.4 \pm 5.5$ & \\
\hline & III & $27.4 \pm 1.8$ & $26.1 \pm 5.8$ & $19.8 \pm 1.1$ & $22.9 \pm 2.3$ & & $24.2 \pm 4.6$ & $23.9 \pm 4.2$ & \\
\hline & $\mathrm{Av}$ & $19.84 \pm 1.1 \mathrm{a}$ & $19.53 \pm 3.3 \mathrm{a}$ & $13.58 \pm 0.6 \mathrm{c}$ & $17.05 \pm 1.1 \mathrm{~b}$ & $<0.01$ & $17.20 \pm 2.5 \mathrm{a}$ & $17.80 \pm 3.7 \mathrm{a}$ & 0.228 \\
\hline \multirow[t]{4}{*}{ RS (\%) } & I & $12.4 \pm 0.8$ & $14.0 \pm 1.1$ & $12.1 \pm 1.6$ & $10.6 \pm 2.0$ & & $12.2 \pm 2.2$ & $12.4 \pm 1.5$ & \\
\hline & II & $12.6 \pm 1.2$ & $11.3 \pm 1.7$ & $11.5 \pm 1.2$ & $11.6 \pm 1.7$ & & $11.8 \pm 1.3$ & $11.7 \pm 1.7$ & \\
\hline & III & $15.5 \pm 1.5$ & $14.7 \pm 1.8$ & $14.6 \pm 1.1$ & $12.2 \pm 1.5$ & & $14.8 \pm 1.9$ & $13.7 \pm 1.7$ & \\
\hline & $\mathrm{Av}$ & $13.50 \pm 0.7 \mathrm{a}$ & $13.33 \pm 0.9 \mathrm{a}$ & $12.74 \pm 1.0 \mathrm{a}$ & $11.44 \pm 0.9 \mathrm{~b}$ & $<0.01$ & $12.94 \pm 1.3 \mathrm{a}$ & $12.57 \pm 1.1 \mathrm{a}$ & 0.210 \\
\hline
\end{tabular}

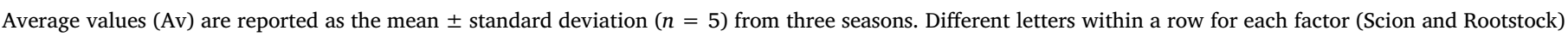

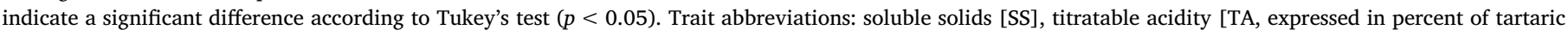
acid], ratio soluble solids/titratable acidity [SS/TA], reducing sugars [RS, expressed in percent of glucose].

1 I, II and III: measurements performed at first, second and third seasons, respectively.

Thus, the highest yield and productivity were obtained from varieties 'Isabel Precoce' and IAC 138-22 'Máximo', with an average of $2.76 \mathrm{~kg}$ / vine and $12.5 \mathrm{tha}^{-1}$, respectively. Compared with vineyards producing wine grapes, the yield of these juice grape varieties was sizeable. The average yield for wine grape varieties is usually in the range of 5 to $15 t$ $\mathrm{ha}^{-1}$, depending on vine density and cultural practices (FAO and OIV, 2017).

Under subtropical conditions, Hernandes et al. (2010) recorded higher yields for IAC 138-22 'Máximo' than the values reported in this study for the same variety, with an average of $4.28 \mathrm{~kg} /$ vine and 22.5 bunches per vine. The increase in yield can be explained by the greater bunch mass found by those authors (195.8 versus $134 \mathrm{~g}$ ). However, the productivity of 'Isabel Precoce' in the present study was higher than that found for the variety in temperate climate conditions, which produced $6.71 \mathrm{t} \mathrm{ha}^{-1}$ (Botelho et al., 2011).

The 'BRS Carmem' variety presented low yield and productivity at $1.45 \mathrm{~kg} / \mathrm{plant}$ and $6.6 \mathrm{t} \mathrm{ha}^{-1}$, respectively. This result was related to the low number of bunches per vine, which might be related to several factors, e.g., difficulty in budburst and low bud fruitfulness in the first few nodes at the base of the shoots. To standardize treatments, all vines were cane pruned to leave one to two nodes. According to Camargo et al. (2008), 'BRS Carmem' vines should be pruned to leave six to eight nodes because the first ones are less fruitful. Thus, the pruning management of this variety must be adjusted to increase its potential yield.

Regarding the effect of the rootstocks on yield components, vines grafted onto 'IAC 766' showed higher number of bunches, yield, and productivity than those grafted onto 'IAC 572'. These results might be related to the different levels of vigor conferred by rootstocks to scions, because 'IAC 572' is more vigorous than 'IAC 766' (Maia and Camargo, 2012). In general, particularly in low-nutrition soils, more vigorous rootstocks have a greater capacity for absorption and translocation of water and nutrients that favor vine performance. However, under optimal climate and soil conditions, vigorous rootstocks may transmit excess vegetative vigor to the canopy, negatively affecting their productive characteristics (Alvarenga et al., 2002), which might have occurred in this study.

\subsection{Physicochemical characteristics of the grapes}

Most physical attributes of the grapes were significantly affected by the rootstocks. 'IAC 766' favored increases in mass, length, and width of bunches and berries, compared with those in 'IAC 572' (Table 2). Borges et al. (2014) did not observe significant differences between 'IAC 766' and 'IAC 572' for number of bunches per vine and bunch mass for the 'Concord' variety, which indicates that different varieties respond differently to the use of the same rootstocks. In varieties of $V$. vinifera table grapes, the number of berries per bunch, berry diameter, berry mass and rachis mass showed great variability using the same rootstocks (Ibacache et al., 2016).The level of vigor conferred by different rootstocks to scions may be a fundamental factor in changing the physical attributes of grapes (Bascunán-Godoy et al., 2017). However, the physiological and/or biochemical interactions that occur between scion and rootstock capable of providing these effects remain unclear.

Regarding scion varieties, 'BRS Cora' and IAC 138-22 'Máximo' had the largest bunches. Nevertheless, the heaviest bunches were obtained from 'BRS Carmem' and IAC 138-22 'Máximo'. The greater bunch mass of 'BRS Carmem' than that of 'BRS Cora' was primarily due to the number of berries per bunch of these varieties, because these variables were significantly correlated $(r=0.98, p<0.01)$. However, the highest number of berries per bunch was found in IAC 138-22 'Máximo'. As observed in this study, the number of berries can vary widely among different grape varieties as a genetically controlled trait. However, the characteristic is strongly influenced by the berry percentage set (Rizzon and Miele, 2002), which can vary considerably.

The number of berries per bunch was significantly negatively correlated with mass $(r=-0.91, p<0.01)$, length $(r=-0.87, p<$ $0.01)$ and width $(r=-0.87, p<0.01)$ of the berries. Thereby, the smallest berry mass, length and width $(1.56 \mathrm{~g}, 1.48 \mathrm{~cm}$ and $1.30 \mathrm{~cm}$, respectively) were from IAC 138-22 'Máximo'. By contrast, 'Isabel Precoce' and 'BRS Cora' had larger and heavier berries. Berry size in a grape variety is also a genetically controlled character and normally does not vary much within a known variety. However, other factors may influence berry size; for example, the number of seeds per berry, because fruit development is regulated through hormones produced 
Table 4

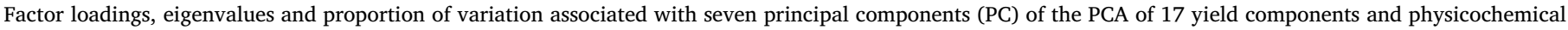
traits in eight scion-rootstock grapevine combinations.

\begin{tabular}{|c|c|c|c|c|c|c|c|}
\hline Traits & PC1 & PC2 & PC3 & PC4 & PC5 & PC6 & PC7 \\
\hline NBchV & -0.075 & -0.521 & 0.843 & -0.097 & -0.051 & 0.029 & -0.005 \\
\hline YldV & -0.102 & -0.408 & 0.855 & 0.298 & -0.059 & -0.010 & -0.007 \\
\hline Pdt & -0.102 & -0.408 & 0.855 & 0.298 & -0.059 & -0.010 & -0.007 \\
\hline BchM & -0.207 & 0.075 & -0.243 & 0.914 & 0.239 & 0.016 & -0.006 \\
\hline BchL & -0.187 & -0.771 & -0.473 & 0.316 & -0.196 & -0.076 & -0.053 \\
\hline BchW & -0.096 & -0.817 & -0.509 & 0.175 & -0.154 & 0.084 & 0.054 \\
\hline NBryBch & -0.931 & 0.013 & -0.213 & 0.296 & 0.012 & -0.021 & 0.021 \\
\hline RchM & -0.958 & -0.041 & 0.112 & 0.244 & 0.088 & -0.032 & 0.015 \\
\hline RchM/BchM & -0.965 & -0.079 & 0.203 & -0.134 & 0.050 & 0.027 & 0.010 \\
\hline BryM & 0.984 & 0.082 & 0.109 & 0.109 & 0.042 & -0.004 & -0.014 \\
\hline BryL & 0.988 & 0.002 & -0.054 & 0.137 & 0.033 & 0.014 & -0.005 \\
\hline BryW & 0.968 & 0.192 & 0.056 & 0.136 & 0.056 & -0.028 & -0.027 \\
\hline $\mathrm{pH}$ & -0.614 & 0.660 & -0.334 & 0.138 & -0.234 & 0.018 & -0.030 \\
\hline SS & 0.968 & -0.107 & -0.030 & 0.167 & -0.069 & -0.111 & 0.072 \\
\hline TA & 0.527 & -0.759 & -0.375 & -0.064 & 0.015 & -0.011 & -0.020 \\
\hline SS/TA & -0.134 & 0.911 & 0.277 & 0.199 & -0.175 & -0.072 & 0.014 \\
\hline RS & 0.780 & 0.423 & 0.136 & 0.395 & -0.140 & 0.134 & 0.006 \\
\hline Eigenvalue & 7.929 & 3.955 & 3.179 & 1.614 & 0.255 & 0.053 & 0.014 \\
\hline Variability (\%) & 46.640 & 23.266 & 18.701 & 9.495 & 1.500 & 0.313 & 0.085 \\
\hline Cumulative (\%) & 46.640 & 69.906 & 88.607 & 98.101 & 99.601 & 99.915 & 100.000 \\
\hline
\end{tabular}

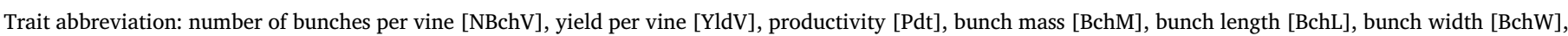

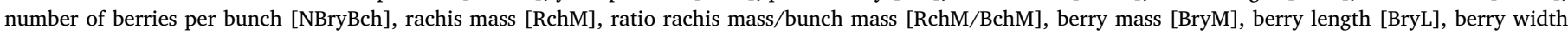
[BryW], soluble solids [SS], titratable acidity [TA], ratio soluble solids/titratable acidity [SS/TA], reducing sugars [RS].

A
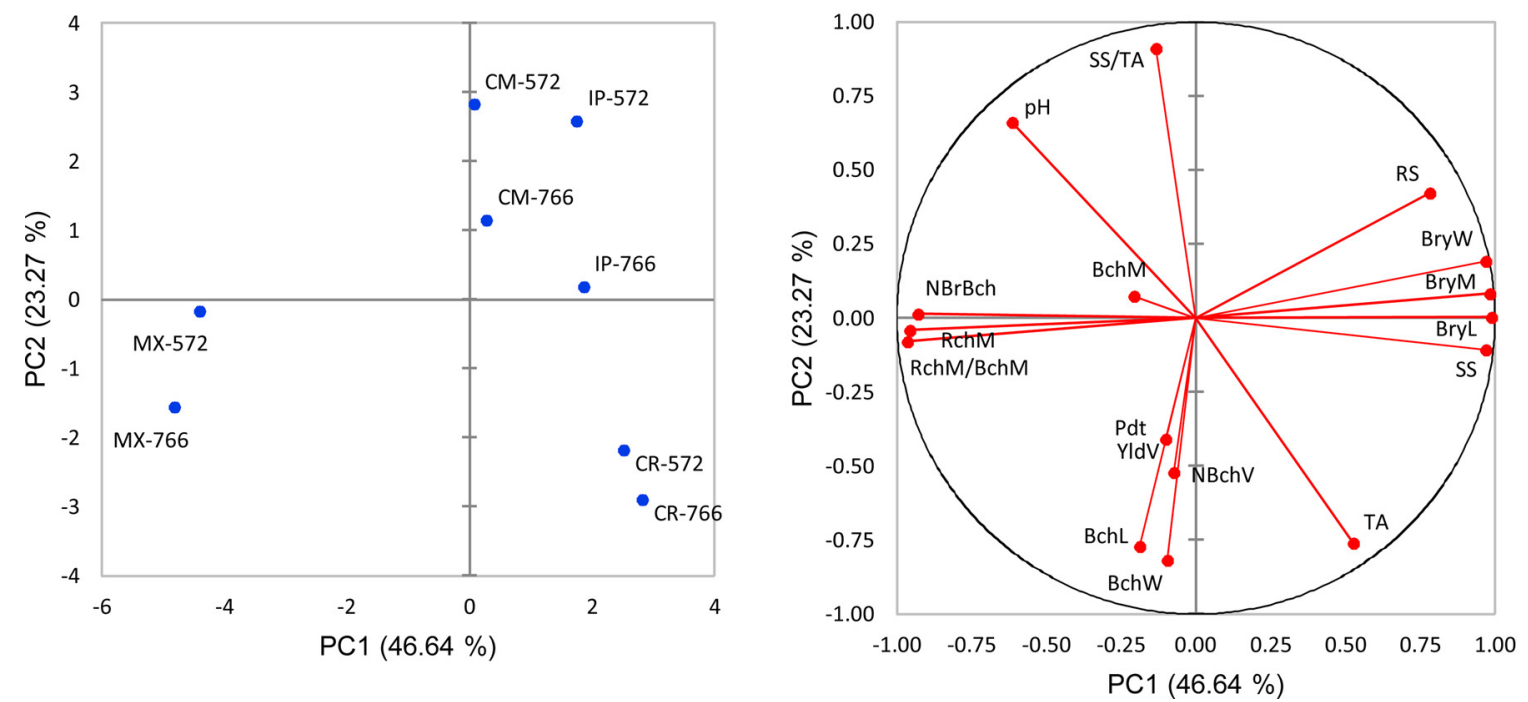

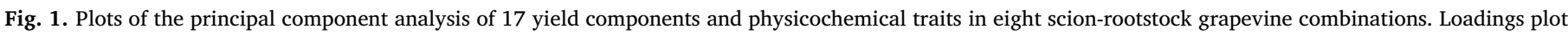
(A) and scores plot (B). See Table 4 for trait labels.

from the seed (Sabir, 2015).

Significant differences were observed among the scion varieties regarding the chemical characteristics of grape must (Table 3 ). The $\mathrm{pH}$ ranged from 3.17 to 3.33 . These values are considered adequate in grapes for processing. $\mathrm{pH}$ values ranging between 3.2 and 3.4 are required, because this characteristic is directly related to the stability of anthocyanins and the color intensity of grape juice or red wine (Yamamoto et al., 2015).

The 'BRS Cora' and 'Isabel Precoce' grapes presented the highest soluble solids (SS) contents, followed by 'BRS Carmem' grapes (15.9, 15.4 and $15.1^{\circ}$ Brix, respectively). Based on Brazilian regulation (Brasil, 2000 ), the minimum SS content for juice grapes is $14.0^{\circ}$ Brix; thus, the SS averages obtained in this study for 'BRS Cora', 'Isabel Precoce' and 'BRS Carmem' grapes were above the established standard. Nevertheless, the IAC 138-22 'Máximo' grape showed limited sugar accumulation potential, averaging $13.5^{\circ}$ Brix, which might be related to a genetic deficiency. Similar results are reported by others (Pedro Júnior et al., 2014).

The grape varieties 'BRS Cora', 'Isabel Precoce' and 'BRS Carmem' presented the highest reducing sugar (RS) content. A positive correlation was observed between SS and RS content $(r=0.77, p<0.01)$. In fact, small amounts of sucrose and other carbohydrates may be present in the grape. However, the reducing sugars glucose and fructose are the primary sugars and constitute the major part of the soluble solids content of must (Conde et al., 2007; González-Fernández et al., 2012).

Regarding titratable acidity (TA), similar results were found for 'Isabel Precoce', 'BRS Carmem' and IAC 138-22 'Máximo', with an average of $0.87 \%$ tartaric acid. This value is below the maximum limit established by Brazilian regulation of 0.90\% (Brasil, 2000). However, the 'BRS Cora' grape presented a titratable acidity average $(1.30 \%)$ 
above that limit. Because the maturation index is derived from the balance between sugars and organic acids (SS/TA), even with high soluble solids content, the high titratable acidity obtained in the 'BRS Cora' provided a low SS/TA ratio. For the 'BRS Cora' grape, the characteristic of maintaining high levels of soluble solids and titratable acidity simultaneously, even at full maturation, is also reported by others (Ribeiro et al., 2012).

The rootstocks had a significant effect on most chemical attributes of the grapes. Compared with 'IAC 572', 'IAC 766' provided lower $\mathrm{pH}$ and higher contents of soluble solids and titratable acidity for the grapes. In contrast to the results found in this study, 'IAC 766' and 'IAC 572' did not differ in affecting chemical attributes ( $\mathrm{pH}, \mathrm{SS}$, TA and SS/ TA) of Concord grapes (Borges et al., 2014). Thus, the performance of rootstocks is not always uniform, and rootstocks must be tested for each cultivar and location. Studies show that the rootstock can modify leaf photosynthesis of the scions (Koundouras et al., 2008), which is related to the primary and secondary metabolites of the vines and consequently, the chemical quality of the grapes (Dias et al., 2012; Lee and Steenwerth, 2013).

\subsection{Principal components analysis}

Principal components analysis (PCA) was performed on the data set of eight scion-rootstock combinations and 17 traits to provide overview visualization in a reduced dimension. Table 4 summarizes the results of the PCA. The total variability was explained by seven principal components (PC). Of these PCs, the first two (PC1 and PC2) accounted for $69.61 \%$ of the total variation.

The PC1, which explained $46.64 \%$ of the total variation, was effective in separating the IAC 138-22 'Máximo' from the other varieties, primarily from the 'Isabel Precoce' and 'BRS Cora'. The 'BRS Carmem' was centered on the PC1 axis. All varieties were grouped for the two 'IAC 766' and 'IAC 572' rootstocks (Fig. 1A). The examination of PC1 loadings (Fig. 1B) suggested that the separation was due to BryM, BryL, BryW, SS and RS and to NBryBch, RchM, and RchM/BchM with high negative loadings. PC1 scores and loadings suggested that 'Isabel Precoce' and 'BRS Cora' presented larger and heavier berries and higher sugar content than those of the other varieties. However, IAC 138-22 'Máximo' showed a greater number of berries per bunch and rachis mass than those of the others.

The PC2 accounted for $23.27 \%$ of the total variation and was effective in separating 'BRS Carmem' and 'Isabel Precoce' from IAC 13822 'Máximo' and 'BRS Cora'. The most important variables contributing to the PC2 were SS/TA with strong positive loading and BchL, BchW, and TA with high negative loadings. PC2 scores and loadings indicated that the 'BRS Cora' presented larger bunches and grapes with higher acidity content and lower SS/TA ratio than those of 'Isabel Precoce' and 'BRS Carmem'.

The variables related to the yield, i.e., NBchV, YldV, and Pdt, were not effective in separating the scion-rootstock combinations on the first two principal components, certainly because of the similarity among the varieties 'Isabel Precoce', 'BRS Cora' and IAC 138-22 'Máximo' in relation to yield components (Table 1 ). Table 4 shows that these variables were correlated with the third principal component (PC3), with strong positive loadings, which explained an additional $18.70 \%$ of the total variance. Therefore, the principal components analysis was efficient in confirming the results presented in this work.

\section{Conclusions}

The varieties 'Isabel Precoce', 'BRS Cora' and IAC 13822 'Máximo' presented high fruit yield, with number of bunches and yield per vine similar to one another and superior to those of 'BRS Carmem'. Physicochemical grape characteristics were significantly different among the varieties. 'Isabel Precoce' and 'BRS Carmem' grapes had balanced levels of sugar and acid content. 'BRS Cora' presented large bunches and berries, reaching high soluble solids contents, despite the high acidity. IAC 138-22 'Máximo' grape also had large bunches but had small berries and limited potential in the accumulation of sugars. The 'IAC 766' rootstock resulted in the best performance across all four varieties evaluated, showing the maximum results in terms of fruit yield and physicochemical quality attributes of grapes.

\section{Conflict of interest}

The authors declare that they have no conflict of interest.

\section{Acknowledgments}

This work was supported by the Conselho Nacional de Desenvolvimento Científico e Tecnológico (CNPq, process n 140211/ 2015-2); and the Fundação de Amparo à Pesquisa do Estado de São Paulo (FAPESP, process $n^{\circ} 2015 / 16440-5$ ). The authors are grateful to Centro Avançado de Pesquisa Tecnológica do Agronegócio de Seringueira e Sistemas Agroflorestais for the availability of the experimental area where this work was performed.

\section{References}

Alvarenga, Â.A., Regina, M.A., Fráguas, J.C., Chalfun, N.N.J., Silva, A.L., 2002. Influência do porta-enxerto sobre o crescimento e produção da cultivar de videira Niágara Rosada (Vitis labrusca L. x Vitis vinifera L.), em condições de solo ácido. Ciênc. Agrotec. Ed. Esp. 1459-1464.

Bascunán-Godoy, L., Franck, N., Zamorano, D., Sanhueza, C., Carvajal, D.E., Ibacache, A., 2017. Rootstock effect on irrigated grapevine yield under arid climate conditions are explained by changes in traits related to light absorption of the scion. Sci. Hortic. 218 284-292. http://dx.doi.org/10.1016/j.scienta.2017.02.034.

Borges, Rde S., Roberto, S.R., Yamashita, F., de Assis, A.M., Yamamoto, L.Y., 2014. Produção e qualida de de frutos de clones de vi deira 'Concord' sobre diferentes portaenxertos. Pesq. Agropec. Trop. 44, 198-204.

Botelho, R.V., Pavanello, A.P., Pires, E.J.P., Terra, M.M., Müller, M.M.L., 2011. Organic production of red wine grapes under plastic cover in subtropical region of Brasil. Ciênc. Agrotec. 35, 1186-1195.

Brasil, 2000. Instrução Normativa $n^{\circ}$ 01, de 07 de janeiro de 2000. Regulamento técnico geral para fixação dos padrões de identidade e qualidade para polpa de fruta. Diário Oficial da República Federativa do Brasil, Brasília, DF, pp. 54-58 10 jan. 2000, Seção 1.

Camargo, U.A., 2004. Isabel Precoce: alternativa para a vitivinicultura brasileira. Comun. Técnico [da] Embrapa Uva e Vinho 54, 1-6. http://www.infoteca.cnptia.embrapa. br/infoteca/handle/doc/539514 (Accessed 05.09.17).

Camargo, U.A., Maia, J.D.G., 2004. 'BRS Cora'. Nova cultivar de uva para suco, adaptada a climas tropicais. Comun. Técnico [da] Embrapa Uva e Vinho 53, 1-7. http://www. infoteca.cnptia.embrapa.br/infoteca/handle/doc/539513 (Accessed 05.09.17).

Camargo, U.A., Maia, J.D.G., Ritschel, P.S., 2008. 'BRS Carmem'. Nova cultivar de uva tardia para suco. Comun. Técnico [da] Embrapa Uva e Vinho 84, 1-8. http://www. infoteca.cnptia.embrapa.br/infoteca/handle/doc/542548 (Accessed 05.25.17).

Camargo, U.A., Mandelli, F., Conceição, M.A.F., Tonietto, J., 2012. Grapevine performance and production strategies in tropical climates. Asian J. Food Agro-Ind. 5, 257-269.

Conceição, M.A.F., Tecchio, M.A., Souza, R.T., Silva, M.J.R., Moura, M.F., 2017. Estimativa dos coeficientes de cultivo (kc) de videiras para suco. Agrometeoros 25, 203-210.

Conde, C., Silva, P., Fontes, N., Dias, A.C.P., Tavares, R.M., Sousa, M.J., Agasse, A., Delrot, S., Gerós, H., 2007. Biochemical changes throughout grape berry development and fruit and wine quality. Food 1, 1-22.

Dias, F.A.N., Mota, R.V., Fávero, A.C., Purgatto, E., Shiga, T.M., Souza, C.R., Pimentel, R.M.A., Regina, M.A., 2012. Videira 'Syrah' sobre diferentes porta-enxertos em ciclo de inverno no sul de Minas Gerais. Pesq. Agrop. Bras. 47, 208-215.

Embrapa, 2006. Sistema Brasileiro de Classificação de Solos. Centro Nacional de Pesquisa de Solos. Embrapa Solos, Rio de Janeiro p. 306.

FAO, OIV, 2017. Table and dried grapes: world data available. FAO-OIV Focus 2016. http://www.oiv.int/js/lib/pdfjs/web/viewer.html?file=/public/medias/5268/faooiv-focus-2016.pdf (Accessed 03.09.18).

González-Fernández, A.B., Marcelo, V., Valenciano, J.B., Rodríguez-Pérez, J.R., 2012. Relationship between physical and chemical parameters for four commercial grape varieties from the Bierzo region (Spain). Sci. Hortic. 147, 111-117. http://dx.doi. org/10.1016/j.scienta.2012.09.009.

Granato, D., Carrapeiro, M.M., Fogliano, V., Van Ruth, S.M., 2016. Effects of geographical origin, varietal and farming system on the chemical composition and functional properties of purple grape juices: a review. Trends Food Sci. Technol. 52, 31-48. http://dx.doi.org/10.1016/j.scienta.2016.03.040.

Hernandes, J.L., Pedro Júnior, M.J., Santos, A.O., Tecchio, M.A., 2010. Fenologia e produção de cultivares americanas e híbridas de uvas para vinho, em Jundiaí-SP. Rev. Bras. Frutic. 32, 135-142.

Ibacache, A., Albornoz, F., Zurita-Silva, A., 2016. Yield responses in Flame seedless, 
Thompson seedless and Red Globe table grape cultivars are differentially modified by rootstocks under semi arid conditions. Sci. Hortic. 204, 25-32. http://dx.doi.org/10. 1016/j.scienta.2016.03.040.

Jin, Z., Sun, T., Sun, H., Yue, Q., Yao, Y., 2016. Modifications of 'Summer Black' grape berry quality as affected by the different rootstocks. Sci. Hortic. 210, 130-137. http://dx.doi.org/10.1016/j.scienta.2016.07.023.

Koundouras, S., Tsialtas, I.T., Zioziou, E., Nikolaou, N., 2008. Rootstock effects on the adaptive strategies of grapevine (Vitis vinifera L. cv. Cabernet-Sauvignon) under contrasting water status: leaf physiological and structural responses. Agric. Ecosyst. Environ. 128, 86-96. http://dx.doi.org/10.1016/j.agee.2008.05.006.

Lee, J., Steenwerth, K.L., 2013. 'Cabernet Sauvignon' grape anthocyanin increased by soil conservation practices. Sci. Hortic. 159, 128-133. http://dx.doi.org/10.1016/j. scienta.2013.05.025.

Lima, M.S., Silani, I.S.V., Toaldo, I.M., Corrêa, L.C., Biasoto, A.C.T., Pereira, G.E., Bordignon-Luiz, M.T., Ninow, J.L., 2014. Phenolic compounds, organic acids and antioxidant activity of grape juices produced from new Brazilian varieties planted in the northeast region of Brazil. Food Chem. 161, 94-103. http://dx.doi.org/10.1016/ j.foodchem.2014.03.109.

Maia, J.D.G., Camargo, U.A., 2012. O cultivo da videira Niágara no Brasil, 1st ed. Empresa Brasileira de Pesquisa Agropecuária, Brasília, DF, Embrapa.

Mello, L.M.R., 2017. Vitivinicultura brasileira: panorama 2016. Comun. Técnico [da] Embrapa Uva e Vinho 199, 1-7. http://www.infoteca.cnptia.embrapa.br/infoteca/ handle/doc/1086551 (Accessed 03.23.18).

Nelson, N.A., 1944. A photometric adaptation of Somogyi method for the determination of glucose. J. Biol. Chem. 135, 136-175.

OIV - Organisation Internationale de la Vigne et du Vin, 2011. Recueil des methods internationals d'analyse des vins et des moûts. Methods Anal. Edition 2011. 8th Assemblée Générale, 21 June 2010, Paris.

Pedro Júnior, M.J., Hernandes, J.L., Blain, G.C., Bardin-Camparotto, L., 2014. Curva de maturação e estimativa do teor de sólidos solúveis e acidez total em função de grausdia: Uva IAC 138-22 'Máximo'. Bragantia 73, 81-85.

Ribeiro, T.P., de Lima, M.A.C., Alves, R.E., 2012. Maturação e qualida de de uvas para suco em condições tropicais, nos primeiros ciclos de produção. Pesq. Agropec. Bras. 47, 1057-1065.

Rizzon, L.A., Miele, A., 2002. Avaliação da cv. Cabernet Sauvignon para elaboração de vinho tinto. Ciênc. Tecnol. Alim. 22, 192-198.

Sabir, A., 2015. Xenia and metaxenia in grapes: differences in berry and seed characteristics of maternal grape cv. 'Narince' (Vitis vinifera $\mathrm{L}$.) as influenced by different pollen sources. Plant Biol. 17, 567-573. http://dx.doi.org/10.1111/plb.12266.

Silva, M.J.R., Vedoato, B.T.F., Lima, G.P.P., Moura, M.F., Coser, G.M.A.G., Watanabe, C.Y., Tecchio, M.A., 2017. Phenolic compounds and antioxidant activity of red and white grapes on different rootstocks. Afr. J. Biotechnol. 16, 664-671. http://dx.doi. org/10.5897/AJB2016.15837.

Vrsic, S., Pulko, B., Kocsis, L., 2015. Factors influencing grafting success and compatibility of grape rootstocks. Sci. Hortic. 181, 168-173. http://dx.doi.org/10.1016/j. scienta.2014.10.058.

Yamamoto, L.Y., Assis, A.M., Roberto, S.R., Bovolenta, Y.R., Nixford, S.L., García-Romero, E., Gómez-Alonso, S., Hermanoín-Gutiérrez, I., 2015. Application of abscisic acid (S $-\mathrm{ABA}$ ) to cv. Isabel grapes (Vitis vinifera $\times$ Vitis labrusca) for color improvement: effects on color, phenolic composition and antioxidant capacity of their grape juice. Food Res. Int. 77, 572-583. http://dx.doi.org/10.1016/j.foodres.2015.10.019. 